\title{
Upregulated Expression and Function of Integrin Adhesive Receptors in Systemic Lupus Erythematosus Patients with Vasculitis
}

\author{
Tsutomu Takeuchi, Kouichi Amano, Hiromi Sekine, Jun Koide, and Tohru Abe \\ Department of Internal Medicine, Saitama Medical Center, Saitama Medical School, \\ Saitama 350, Japan
}

\begin{abstract}
Upregulation of integrin adhesive receptors has been implicated in various pathological conditions. We examined expression and function of integrin adhesive receptors on peripheral blood lymphocytes from patients with systemic lupus erythematosus (SLE), particularly those with the complication of vasculitis, and found that VLA-4 and LFA-1 expression was increased in SLE patients with vasculitis, while LFA-1 but not VLA-4 expression was increased in those without vasculitis. These results suggested a role of VLA-4 in the pathogenesis of vasculitis in SLE. Functional studies further demonstrated that adhesion to cytokine-activated human umbilical cord vein endothelial cells and to the CS-1 alternatively spliced domain of fibronectin was significantly increased in SLE patients with vasculitis. Analysis of the functional epitopes on the $\alpha_{4}$ chain demonstrated that antigen densities of all the functional epitopes were increased in those with vasculitis, indicating that the increased expression of VLA-4 resulted from the increased number of VLA-4 molecules, and was not secondary to an increase in one particular functional epitope. Immunoprecipitation studies further support these results. Interestingly, high molecular weight bands associated with VLA-4 were observed in about half of the SLE patients with vasculitis. These results introduce a possibility that upregulation of integrin adhesive receptors has a potential role in the pathogenesis of vasculitis in SLE. (J. Clin. Invest. 1993. 92:3008-3016.) Key words: systemic lupus erythematosus • integrins • VLA-4 - LFA-1 • vasculitis
\end{abstract}

\section{Introduction}

Systemic lupus erythematosus (SLE) is a multi-system disorder characterized by the production of a variety of autoantibodies (1). Some autoantibodies are pathogenic (2) and cause tissue damage by directly reacting with the surface of platelets and erythrocytes (3), or by forming immune complexes upon

Address correspondence to Dr. Tsutomu Takeuchi, Department of Internal Medicine, Saitama Medical Center, Saitama Medical School, 1981 Tsujido-machi, Kamoda, Kawagoe-shi, Saitama 350, Japan. 1993

Received for publication 15 April 1993 and in revised form 12 July

1. Abbreviations used in this paper: ECM, extracellular matrix; HUVEC, human umbilical cord vein endothelial cell; KLH, keyhole limpet hemocyanin; MFI, mean fluorescence intensity.

J. Clin. Invest.

(C) The American Society for Clinical Investigation, Inc.

0021-9738/93/12/3008/09 \$2.00

Volume 92, December 1993, 3008-3016 activation of complement (4). Anti-DNA and DNA immune complexes have been eluted from the affected kidneys in SLE patients (5), and can cause lupus glomerulonephritis, indicating that immune complex-mediated tissue injuries are of central importance in the pathogenesis of lupus nephritis. This is the best understood scenario for the pathogenesis of SLE. Although the deposition of immune complexes in the tissue has been implicated in the pathogenesis of SLE, it is still possible to assume additional and alternative mechanisms for the tissue injuries in the disease. In this regard, immunopathological findings by Savage (6) showed that immune complexes can rarely be found in the blood vessels from biopsy specimens of SLE patients with vasculitis.

Recently, Phillip et al. suggested that an alternative mechanism of vascular damage may occur in SLE involving intravascular activation and aggregation of neutrophils in the absence of immune complex deposition (7). They introduced the possibility that the Schwartzman reaction is a model of this type of vascular injury, and that it is not mediated by immune complex deposition. Cytokine-primed neutrophil-dependent vascular damage has thus been demonstrated to participate in a rabbit model of hemorrhagic vasculitis in the absence of immune complex deposition (8). If this is the case for the mechanism of tissue injury in SLE, interaction between leukocytes and endothelium or substitium in connective tissues may have a major role in pathogenesis of the disease.

Recent progress in the study of adhesion molecules has revealed that several sets of surface molecules participate in cellcell and cell-substitium adhesions (9). Members of the $\beta 1$ integrin family of adhesion molecules, which comprises nine different $\alpha$ chains and a noncovalently associated $\beta 1$ subunit, are major adhesive receptors for the extracellular matrix (ECM) ${ }^{1}$ proteins (10). On the other hand, the $\beta 2$ integrin family mediates cell-cell adhesion. In the $\beta 1$ integrin subfamily, VLA-4, and now VLA-3, are exceptionally involved in both cell-cell and cell-ECM adhesion. The function and expression of these adhesive receptors are properly regulated (9-11). Upregulation of these adhesive receptors has been found in several pathological conditions such as atherosclerosis (12), and bronchial asthma, as well as in the synovial $\mathrm{T}$ cells of rheumatoid arthritis (13). We also experienced a case of SLE complicated by necrotizing vasculitis of the mesenteric artery, which resulted in perforation of the ileum. In this particular patient, the expression and function of VLA-4 was increased during the vasculitic event, indicating a role of the integrin adhesive receptors in the pathogenesis of vasculitis in SLE. ${ }^{2}$ A similar idea came from

2. Takeuchi, T., K. Aoki, J. Koide, H. Sekine, and T. Abe. 1993. Upregulated expression and function of SM-27 (VLA-4) antigen on T cells from a patient with lupus erythematosus complicated by vasculitis. Manuscript submitted for publication. 
the following studies in which we conducted a series of experiments to identify the surface structures important for the pathogenesis of SLE, and attempted to develop mAbs directed against the surface antigen on lymphocytes from SLE patients on the basis of increased reactivity with the patients' lymphocytes compared to normal individuals. Among those mAbs, we found that a clone, SM-27, recognizing the surface antigen whose expression on SLE lymphocytes was greater than normal lymphocytes, identifies the VLA-4 antigen (14).

These studies, however, were performed only in a case of SLE complicated by vasculitis, and there has not yet been a determination of the expression and function of integrin adhesive receptors in substantial numbers of SLE patients with vasculitis to permit us to draw some conclusions.

In this study we attempted to delineate the expression and function of these adhesive receptors of the integrin family on peripheral blood lymphocytes in patients with SLE. We demonstrated that expression and function of VLA-4 as well as LFA-1 were upregulated in SLE patients with vasculitis, while LFA-1 but not VLA-4 expression was increased in SLE patients without vasculitis. Moreover, the function of VLA-4 in terms of adhesiveness to fibronectin and cytokine-activated endothelial cells is increased in these patients. Immunoprecipitation studies showing increased 140-, 80-, and 70-kD bands of the VLA-4 $\alpha$ chain in patients with vasculitis confirmed the above results. Surprisingly, we also found that $180-\mathrm{kD}$ extra-bands were observed by immunoprecipitation of VLA-4 from peripheral blood lymphocytes of the vasculitis patients. These results introduce a possible role of integrin adhesive receptors in the pathogenesis of vasculitis in SLE.

\section{Methods}

Patients. The sample population consisted of 27 patients with SLE satisfying the classification criteria proposed by the American Rheumatism Association (15). Disease activity was determined for each patient at the time of blood drawing by two physicians on the basis of multi-system disease manifestations, as described previously (16). Briefly, manifestations included fever, rash, arthritis, serositis, vasculitis, active nephritis, and active central nervous system disease. Patients with two or more manifestations were considered to have substantially active disease. Disease duration was determined from the date of diagnosis to that of blood drawing.

Patients with known renal disease were those with hematuria ( $\geq 10$ red blood cells per high power field), proteinuria ( $\geq \lg$ per $24 \mathrm{~h}$ ), RBC casts, or cellular casts, either currently or in the past. Six patients were judged to have vasculitis by demonstration of necrotizing vasculitis in the biopsy specimens. Their clinical manifestations attributable to vasculitis were digital infarction, skin ulcers, optic nerve neuritis, perforation of the ileum, and mononeuritis multiplex. Nine patients were untreated (eight patients without vasculitis and one with vasculitis). 18 patients were receiving low to intermediate doses of prednisolone ( 5 to $30 \mathrm{mg} / \mathrm{d}$ ), but none of the patients had received cytotoxic drugs during the preceding $6 \mathrm{mo}$ at the time of blood drawing.

The normal control population consisted of 42 healthy individuals with no significant illness.

$m A b s$. Spleen cells from BALB/C mice immunized by three intraperitoneal injections of $1 \times 10^{7}$ PBLs from a patient with SLE complicated by vasculitis (case S.M., 38-yr-old female) were fused with NS-1 myeloma cells at a ratio of 10:1 by a standard procedure, and were distributed into 96 wells with normal spleen cells as feeders. Hybridomas producing antibodies reactive with PBL from the SLE patient were selected by means of indirect immunofluorescence with a cytofluorometer and used for further analysis as described previously (14). The isotype of SM-27 was IgG1. The other anti-VLA-4 antibodies are as follows: HP2/ 1 (IgG1; Immunotech S.A., Luminy, France); L25 (IgG2b; Becton Dickinson Immunocytometry Systems, San Jose, CA); and B-5G10 (IgG1; Upstate Biotechnology, Inc., Lake Placid, NY). Leu 5b (CD2), Leu 4 (CD3), Leu 3(CD4), Leu 2 (CD8), G25.2 (CD11a), Leu 15 (CD11b), and L 130 (CD18) were purchased from Becton Dickinson Immunocytometry Systems. T11 (CD2), T3 (CD3), T4 (CD4), T8 (CD8), $2 \mathrm{H} 4$ (CD45RA), and 4B4 (CD29) were purchased from Coulter Immunology (Hialeah, FL). T2/7 (VLA-1) was purchased from T Cell Sciences (Cambridge, MA). BU15 (CD11c), Gi-9 (VLA-2), and SAM-1 (VLA-5) were obtained from Immunotech S.A. M-kid2 (VLA-3) and GoH3 (VLA-6) were obtained from Upstate Biotechnology, Inc. Anti-ICAM-1 and antiVCAM-1 were obtained from British Bio-technology Ltd. (Abingdon, OX, England).

Immunofluorescence analysis. Cells were incubated with $\mathrm{mAbs}$ for $30 \mathrm{~min}$ on ice, then incubated with a $\mathrm{F}\left(\mathrm{ab}^{\prime}\right)_{2}$ fragment of goat antimouse antibody conjugated with FITC (Tago, Inc., Burlingame, CA) as described previously (16).

The flow cytometer (Becton Dickinson Immunocytometry Systems) was run on fixed laser power $(600 \mathrm{~mW}$ at $488 \mathrm{~nm})$ over the period of the study. Flow cytometric standardization was achieved by running 4.05- $\mu \mathrm{m}$ fluorescent beads (Becton Dickinson Immunocytometry Systems), which exhibited constant light scatter and fluorescence properties. Background fluorescence reactivity was determined with control ascitic fluid obtained from mice immunized with a nonsecreting hybridoma. Control and patient samples were run with the FITC photomultiplier adjusted to a high enough voltage such that the fluorescence histogram derived from the forward angle vs. side scattergated lymphocyte population yielded $2-7 \%$ background autofluorescence intensity in the range reserved for positively FITC-stained cells. All samples were, then, analyzed using the fixed photomultiplier setting. For each sample, 10,000 cells were analyzed on a log fluorescence scale on the flow cytometer. For determination of the surface antigen expression, the mean log FITC-fluorescence channel of the positivelystained cells was determined from a single-parameter histogram.

Isolation of cells. PBL from normal individuals and patients with SLE were separated from heparinized venous blood by Ficoll-Hypaque density gradient centrifugation (Pharmacia LKB Biotechnology, Uppsala, Sweden) as described previously (16). PBL were suspended in medium containing 10\% FCS (Hazelton Biologics, Inc., Lenexa, KS) and were incubated on $1 \%$ serum-coated dishes, and passed over a nylon-wool column to yield a $\mathrm{T}$ cell-enriched population. This $\mathrm{T}$ cell population was more than $92 \%$ reactive with anti-T3 antibody.

ECM proteins and their fragments. Fibronectin, laminin, and collagen types I, II, III, IV were purchased from Sigma Chemical Co. (St. Louis, MO). Oligopeptides corresponding to the VLA-4-binding site of fibronectin were synthesized by an amino acid synthesizer (Biolynx Peptide Synthesizer; Pharmacia LKB Biotechnology), then purified with an FPLA system with a mono- $Q$ column. The oligopeptides GPEILDVPST (CS-1A) and TLPHPNLHGP (CS-1B) were conjugated to keyhole limpet hemocyanin (KLH) by the maleimid method as described in the manufacture's brochure (Pierce Chemical Co., Rockford, IL).

Assays of cell attachment to ECM proteins and HUVECS. For cell attachment to ECM proteins, flat-bottomed 96-well microtiter plates (Costar Corp., Cambridge, MA) were coated with $100 \mu \mathrm{l}$ of $0.1 \mathrm{M}$ $\mathrm{NaHCO}_{3}$ containing $10 \mu \mathrm{g}$ of fibronectin, laminin, collagen, CS-1A, and CS-1 $\mathrm{B}$ oligopeptides for $2 \mathrm{~h}$ at $37^{\circ} \mathrm{C}$ and then coating was blocked with RPMI 1640 medium in the presence of $1 \%$ BSA (Sigma Chemical Co.) for $1 \mathrm{~h}$ at $37^{\circ} \mathrm{C}$. For attachment to cytokine-activated human umbilical cord vein endothelial cells (HUVECs), confluent monolayers of HUVECs activated with recombinant IL-1 $\beta$ and TNF $\alpha$ for 24 h ( $10 \mathrm{U} /$ well; Genzyme, Boston, MA) were prepared in 96-well plates. Cells were suspended in RPMI 1640 medium containing $1 \%$ BSA, and plated in triplicate on ECM protein-coated plates ( $100 \mu \mathrm{l}$ final volume). After 30 -min incubation at $37^{\circ} \mathrm{C}$ for ECM binding assay of 15-min incubation at room temperature for monolayer binding assay, unbound cells were removed by three washings with attachment me- 
dium. Bound cells were fixed with $1.25 \%$ glutaraldehyde in PBS $(0.01$ $\mathrm{M}, \mathrm{pH} 7.4$ ) at $4^{\circ} \mathrm{C}$ overnight, and stained with $4 \%$ trypan blue. Bound cells were counted under a microscope, and the results were expressed as bound cells per $\mathrm{mm}^{2}$ or lysed with $10 \%$ SDS and the $\mathrm{OD}_{590}$ was measured by a Titertek Multiscan plus (Flow Laboratories, Horsham, PA). For antibody blocking assay, $\mathrm{mAbs}$ were added $30 \mathrm{~min}$ before the adhesion assay.

Cell surface labeling and immunoprecipitation. Cells were surface labeled with sulfo-NHS-biotin (Pierce Chemical Co.) as described in the manufacture's brochure, and lysed in the presence of $1 \%$ NP-40 as described previously (17). Cell lysates were precleared with insoluble protein A (Sigma Chemical Co.), and incubated with mAbs. The immune complexes were precipitated with protein G-agarose beads (Pharmacia LKB Biotechnology), and washed five times with lysis buffer. Sample buffer was added, and the mixture was boiled for $5 \mathrm{~min}$. The samples were run on $6 \%$ polyacrylamide gels. After electrophoresis, the gels were equilibrated for $10 \mathrm{~min}$ in a transfer buffer $(25 \mathrm{mM}$ Tris- $\mathrm{HCl}, 192 \mathrm{mM}$ glycine, and $20 \%$ methanol) and proteins were electrophoretically blotted onto polyvinylidene difluoride membranes (Millipore Corp., Bedford, MA). The membranes were soaked at $37^{\circ} \mathrm{C}$ for $1 \mathrm{~h}$ in blocking agents (Blockace; Dainippon Pharmaceuticals, Tokyo), and streptoavidin-horseradish peroxidase conjugate (Amersham International, Amersham, UK) was added at a dilution of 1:1,000 with PBS containing $0.1 \%$ Tween- 20 and $10 \%$ Blockace. After incubation at $37^{\circ} \mathrm{C}$ for $1 \mathrm{~h}$, the membranes were washed three times and the detection of the horseradish peroxidase-labeled proteins was carried out with chemiluminescence-enhancing reagents as described in the manufacture's brochure (Amersham International). The treated membranes were visualized on ECL $\mathrm{x}$-ray films.

\section{Results}

Expression of integrin adhesive receptors in SLE. To delineate a role of integrin adhesive receptors in the pathogenesis of SLE with vasculitis, we examined expression of integrins on PBL in SLE patients with vasculitis, those without vasculitis, and normal individuals. The age, sex, disease activity, disease type, disease duration, clinical manifestations of the vasculitis, and dose of prednisolone in SLE patients studied are summarized in Table I. The mean age and sex were comparable between the SLE patients and normal individuals. All patients without vasculitis had active disease, except for two, while all six patients with vasculitis were judged to be active. Disease type and disease duration were comparable between SLE patients without and with vasculitis. The dose of prednisolone was not statistically significant between these two populations of SLE.

The representative cytofluorographic pattern in Fig. 1 shows that CD29, CD11a, and CD18 antigens have a bimodal distribution in fluorescence intensity on a log scale, indicating that there are two distinct fractions expressing high or low levels of CD29, CD1 1a, and CD18. Therefore, the percent expression and mean fluorescence intensity (MFI) of the population expressing high levels of antigens are shown as CD29 high, CD1 la high, and CD18 high. As seen in Table II, there are no significant changes in percent expression of CD3, VLA-1, VLA-2, VLA-3, VLA-4, VLA-5, and VLA-6, whereas the percent expression of the CD29 high population was increased in the SLE patients with vasculitis, supporting the previous observation. With respect to $\beta 2$ integrins, the percent expressions of CD1 la high, CD11c, and CD18 high are significantly higher in the vasculitis patients than in normal individuals $(56 \pm 29 \%$, $45 \pm 6 \%$, and $57 \pm 29 \%$, respectively), while CD1 la high and CD 18 high are also increased in the SLE patients without vasculitis $(49 \pm 18 \%$, and $47 \pm 19 \%$, respectively). When we determined the antigen densities by comparing the MFI of the surface antigens, that of VLA- $4 \alpha$ was significantly increased in the SLE patients with vasculitis, compared to normal individuals ( $101 \pm 56$, and $61 \pm 22$, respectively), whereas no significant increase was observed in the SLE patients without vasculitis. Similarly, the MFI of CD11a as well as CD18 was increased in the vasculitis patients. These results clearly indicate that expression of VLA-4 and LFA-1 antigens was upregulated in the vasculitis patients. It is well accepted that after activation it is the CD4+ T cells that express adhesion molecules such as CD29,

Table I. Characteristics of SLE Patients Studied

\begin{tabular}{|c|c|c|c|c|c|c|c|c|}
\hline Subject & Number & Age & Sex & Activity & $\begin{array}{l}\text { Disease } \\
\text { subset }\end{array}$ & $\begin{array}{l}\text { Disease } \\
\text { duration }\end{array}$ & $\begin{array}{l}\text { Clinical manifestation } \\
\text { of the vasculitis }\end{array}$ & Prednisolone \\
\hline & & & $(F / M)$ & (active/inactive) & $(R / N R)$ & & & $(m g / d)$ \\
\hline Normal & 42 & $\begin{array}{l}31.8 \pm 6.4 \\
(20 \text { to } 48)\end{array}$ & $42 / 0$ & - & - & - & 一 & - \\
\hline SLE & 21 & $\begin{array}{l}35.5 \pm 12.6 \\
(14 \text { to } 59)\end{array}$ & $21 / 0$ & $19 / 2$ & $4 / 17$ & $\begin{array}{l}21 \pm 12 \\
(3-48)\end{array}$ & - & $\begin{array}{c}8.8 \pm 9.0 \\
(0 \text { to } 30)\end{array}$ \\
\hline SLE with vasculitis & 6 & $\begin{array}{l}31.2 \pm 7.9 \\
(23 \text { to } 41)\end{array}$ & $6 / 0$ & $6 / 0$ & $1 / 5$ & $\begin{array}{l}25 \pm 11 \\
(9-37)\end{array}$ & & $\begin{array}{c}9.8 \pm 4.7 \\
(0-15)\end{array}$ \\
\hline S.M. & & 41 & $\mathrm{~F}$ & Active & NR & & $\begin{array}{l}\text { Digital necrosis } \\
\text { Optic neuritis }\end{array}$ & 10 \\
\hline S.A. & & 37 & $\mathrm{~F}$ & Active & NR & & $\begin{array}{l}\text { Ileal perforation due to } \\
\text { mesenteric vasculitis }\end{array}$ & 10 \\
\hline F.S & & 24 & F & Active & NR & & Digital necrosis & 12 \\
\hline N.Y. & & 23 & $\mathrm{~F}$ & Active & $\mathbf{R}$ & & Skin ulcers & 15 \\
\hline Y.K. & & 38 & 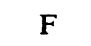 & Active & NR & & Digital necrosis & 0 \\
\hline T.K. & & 24 & F & Active & NR & & $\begin{array}{l}\text { Skin ulcers } \\
\text { Digital necrosis }\end{array}$ & 12 \\
\hline
\end{tabular}

Results were expressed as mean $\pm \mathrm{SD}$. Disease subset was shown as $\mathrm{R}$ (renal) or NR (non-renal). Disease duration was expressed as mean months \pm SD. 


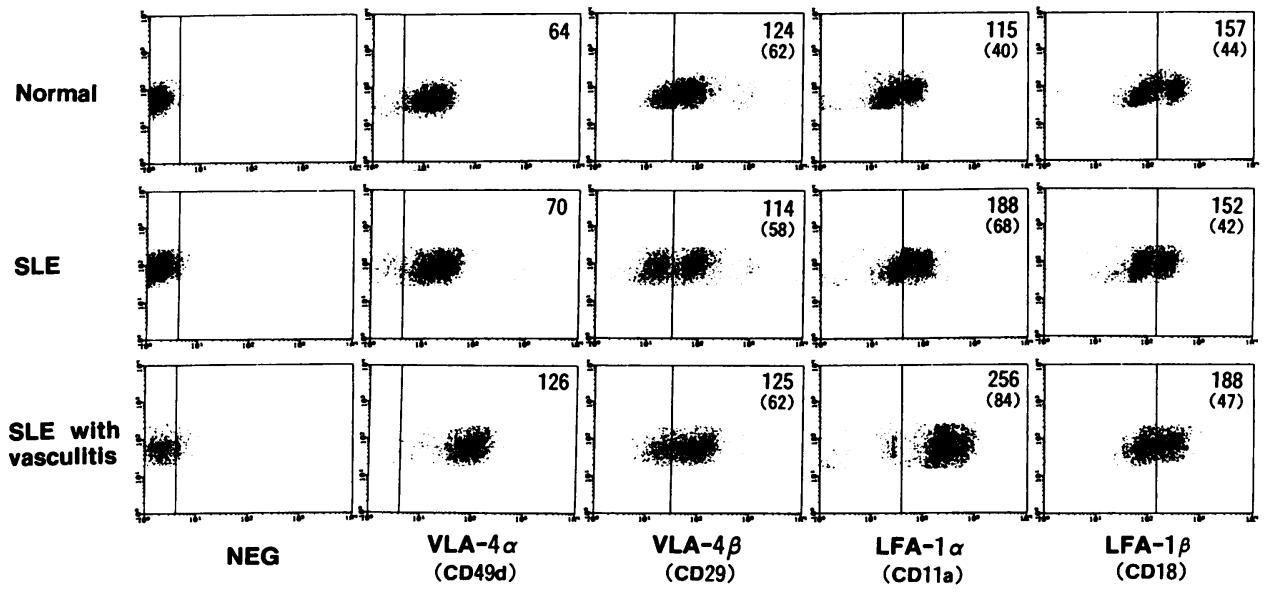

Figure 1. Flow cytometric analysis for the integrin adhesive receptors on PBLs from representative cases of a normal individual, a SLE patient without vasculitis and that with vasculitis. The ordinate represents the forward light scatter and the abscissa represents the log fluorescence intensity.
PgP-1, and LFA-3 accompanied by an isoform change from CD45RA to CD45RO (18). Accordingly, we next determined the expression of these integrins on CD4+ T cells in SLE. As shown in Table III, the percentage of CD4+CD45RA+ cells decreased and that of CD4+CD29high + cells increased in SLE both with and without vasculitis. Although the percent expression of VLA-6 was significantly high in the SLE patients with vasculitis, that of VLA-5 and VLA- 6 was also high in the SLE patients without vasculitis, when compared to that of normal individuals. Antigen densities as determined by the MFI on a flow cytometer have shown that expression of VLA-4 $(\alpha 4)$ was

Table II. Expression of Integrin Adhesive Receptors on PBLs from SLE and Normal Individuals

\begin{tabular}{lccc}
\hline & & \multicolumn{2}{c}{ SLE } \\
\cline { 3 - 4 } Suface antigen & $\begin{array}{c}\text { Normal } \\
(n=42)\end{array}$ & $\begin{array}{c}\text { Vasculitis(-) } \\
(n=21)\end{array}$ & $\begin{array}{c}\text { Vasculitis(+) } \\
(n=6)\end{array}$ \\
\hline \% expression & & & \\
CD2 & $81 \pm 4$ & $82 \pm 10$ & $85 \pm 9$ \\
CD3 & $72 \pm 6$ & $79 \pm 11$ & $75 \pm 15$ \\
CD4 & $41 \pm 5$ & $38 \pm 13$ & $31 \pm 12^{\S}$ \\
CD8 & $26 \pm 6$ & $35 \pm 12$ & $35 \pm 10^{\ddagger}$ \\
VLA-1 $(\alpha 1)$ & $6 \pm 5$ & $8 \pm 10$ & $16 \pm 14$ \\
VLA-2 $(\alpha 2)$ & $4 \pm 3$ & $9 \pm 11$ & $9 \pm 2$ \\
VLA-3 $(\alpha 3)$ & $16 \pm 15$ & $20 \pm 8$ & $22 \pm 9$ \\
VLA-4 $(\alpha 4)$ & $92 \pm 8$ & $93 \pm 7$ & $96 \pm 4$ \\
VLA-5 $(\alpha 5)$ & $54 \pm 14$ & $48 \pm 14$ & $58 \pm 18$ \\
VLA-6 $(\alpha 6)$ & $30 \pm 17$ & $22 \pm 17$ & $31 \pm 12$ \\
CD29 high & $51 \pm 12$ & $58 \pm 15$ & $65 \pm 16^{*}$ \\
CD11a high & $36 \pm 9$ & $49 \pm 18^{\S}$ & $56 \pm 29^{\ddagger}$ \\
CD11b & $24 \pm 7$ & $30 \pm 15$ & $26 \pm 6$ \\
CD11c & $24 \pm 8$ & $29 \pm 12$ & $45 \pm 6^{\ddagger}$ \\
CD18 high & $36 \pm 10$ & $47 \pm 19^{\ddagger}$ & $57 \pm 29^{\ddagger}$ \\
HML-1 $(\alpha$ E/ $\beta 7)$ & 0 & 0 & 0 \\
Mean fluorescence & & & \\
intensity & & & \\
VLA-4 $(\alpha 4)$ & $61 \pm 22$ & $68 \pm 24$ & $101 \pm 56^{\S}$ \\
CD29 high & $125 \pm 78$ & $120 \pm 57$ & $120 \pm 57$ \\
CD11a high & $186 \pm 52$ & $204 \pm 40$ & $259 \pm 42^{\ddagger}$ \\
CD18 high & $218 \pm 46$ & $217 \pm 42$ & $272 \pm 47^{*}$ \\
& & & \\
\hline
\end{tabular}

${ }^{*} P<0.05 .{ }^{\ddagger} P<0.01 .{ }^{\S} P<0.005$. increased in MFI in the SLE patients with vasculitis, while that of CDIla was increased in the SLE patients both with and without vasculitis.

The results showed that VLA-4 antigen expressed on PBL and CD4+ $T$ cells was significantly high in the SLE patients with vasculitis, while LFA-1 expression was increased in the SLE patients both with and without vasculitis.

Adherence of $T$ cells to cytokine-activated HUVECs in $S L E$. The above results clearly show that the integrin adhesive receptors were upregulated on the $\mathrm{PBL}$ as well as on CD4+ $\mathrm{T}$ cells from SLE patients with vasculitis. Next, we attempted to determine the function of these adhesive receptors. First, we studied adhesion to endothelial cells, since this process should be of primary importance for eliciting vascular inflammation $(9,19)$. Through the use of IL- $1 \beta$ - and TNF $\alpha$-stimulated HUVECs, which have already been shown to express ICAM-1 and VCAM-1 antigens (20), the ligands for LFA-1 and VLA-4, respectively, adhesion of peripheral blood $\mathrm{T}$ cells from normal individuals, and patients without, or with vasculitis, was examined. As shown in Fig. 2, T cells from patients with vasculitis showed a significant increase in adhesion to cytokine-activated endothelial cells ( $189 \pm 47$ ), whereas $T$ cells from patients without vasculitis showed a slight but significant increase in adhe-

Table III. Expression of Integrin Adhesive Receptors on CD4+ TCells in SLE Patients with or without Vasculitis

\begin{tabular}{lccc}
\hline & & \multicolumn{2}{c}{ SLE } \\
\cline { 3 - 4 } Subset of CD4 & $\begin{array}{c}\text { Normal } \\
(n=42)\end{array}$ & $\begin{array}{c}\text { Vasculitis(-) } \\
(n=21)\end{array}$ & $\begin{array}{c}\text { Vasculitis(+) } \\
(n=6)\end{array}$ \\
\hline $\begin{array}{l}\text { \% of CD4+ subset } \\
\text { CD45RA+ }\end{array}$ & $39 \pm 10$ & $29 \pm 18^{*}$ & $29 \pm 10^{*}$ \\
CD29 high & $46 \pm 10$ & $61 \pm 26^{*}$ & $71 \pm 31^{*}$ \\
VLA-4 $(\alpha 4)$ & $95 \pm 7$ & $89 \pm 23$ & $97 \pm 12$ \\
VLA-5 $(\alpha 5)$ & $49 \pm 7$ & $68 \pm 11^{\ddagger}$ & $65 \pm 32$ \\
VLA-6 $(\alpha 6)$ & $44 \pm 5$ & $74 \pm 28^{\ddagger}$ & $71 \pm 34^{*}$ \\
MFI & & & \\
VLA-4 $(\alpha 4)$ & $66 \pm 18$ & $66 \pm 78$ & $114 \pm 35^{\ddagger}$ \\
CD29 high & $115 \pm 12$ & $120 \pm 16$ & $125 \pm 20$ \\
CD11a high & $195 \pm 42$ & $235 \pm 48^{*}$ & $270 \pm 81^{*}$ \\
CD18 high & $242 \pm 61$ & $266 \pm 52$ & $259 \pm 29$ \\
\hline
\end{tabular}

${ }^{*} P<0.05 .{ }^{\ddagger} P<0.01$ 
$\mathrm{T}$ cells adhered to cytokine - activated HUVECs (cells $/ \mathrm{mm}^{2}$ )

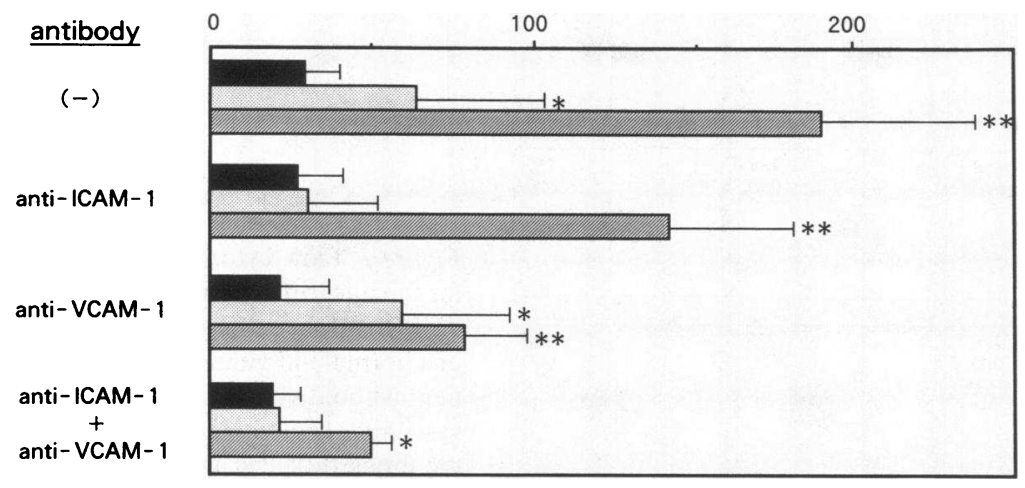

Figure 2. Adhesion of peripheral blood T cells from sexand age-matched normal individuals (black bar; $n=27$ ), SLE patients without vasculitis ( $g r a y$ bar; $n=21$ ), and those with vasculitis ( shaded bar; $n=6$ ) to cytokine-activated HUVECs was examined in the presence of mAbs directed against ICAM-1 and VCAM-1. ${ }^{*} P<0.05,{ }^{* *} P$ $<0.01$. sion $(62 \pm 40)$ and it was only minimal in normal controls (28 \pm 9$)$. Since we detect not only VLA-4/VCAM-1 interaction but also LFA-1 / ICAM-1 interaction in this assay system, we determined the individual pathway by blocking with mAbs to ICAM-1 and/or VCAM1. In the presence of anti-ICAM-1 adhesion of $T$ cells from the SLE patients with vasculitis was decreased to $\sim 75 \%$, but it was still pronounced (142 \pm 46 ). In contrast, adhesion of T cells from the SLE patients without vasculitis was decreased almost the same amount as that of normal individuals $(30 \pm 21$ vs. $26 \pm 13)$. In the presence of anti-VCAM-1, inhibition of adhesion of T cells from the SLE patients with vasculitis was $58 \%$ of that in the absence of blocking antibody, while little inhibition was observed in the SLE patients without vasculitis. The results suggest that the pathways involving VLA-4/VCAM-1 and LFA-1/ICAM-1 were functionally upregulated in the SLE patients with vasculitis, whereas in those without vasculitis only the LFA-1/ICAM-1 pathway was functionally upregulated, supporting the above results obtained with the expression of integrins. Although there was significant inhibition of $T$ cell adhesion to cytokineactivated HUVECs in the presence of anti-ICAM-1 and antiVCAM-1, it is worth noting that the inhibition was only $80 \%$ in the presence of both anti-ICAM-1 and anti-VCAM-1. This implies that additional and/or undefined pathways may contribute to the increased adhesion of $T$ cells of these patients. Nevertheless, VLA-4/VCAM-1 and LFA-1/ICAM-1 seemed to be major pathways for increased adhesion to activated HUVECs in these patients.

Adhesion of peripheral T cells to ECM proteins. It is well known that among $\beta 1$ integrins VLA-4 is a unique molecule that mediates both cell-cell and cell-ECM protein adhesion (21). Therefore, we examined alternative functions of adhesive of VLA-4 to ECM proteins in these patients. For this purpose, we synthesized oligopeptides corresponding to the VLA-4-bind- ing site of fibronectin, which has been shown to be the CS-1 alternatively spliced domain. By using oligopeptides designated CS-1A (GPEILDVPST) as well as nonbinding control peptides designated CS-1B (TLPHPNLHGP), we examined, as depicted in Figs. 3 and 4, adhesion of T cells to these oligopeptides and to three ECM proteins. As shown in Fig. 4, T cells from the representative patient with vasculitis showed pronounced adhesion not only to fibronectin but also to the CS-1 A oligopeptides, while they adhered only slightly to laminin, and adherence to the negative control, BSA, was minimal. Such enhanced adhesion was not observed in $\mathrm{T}$ cells from normal individuals and from the SLE patient without vasculitis. Fig. 3 confirmed the above finding that $\mathrm{T}$ cells from the patients with vasculitis showed a significant increase in adhesion to fibronectin and CS-1A oligopeptides, while adhesion to CS-1B and laminin was only slightly increased. On the other hand, we observed a slight increase in adhesion to fibronectin, CS-1A, and CS-1 B in SLE without vasculitis, but the increase was not significant in comparison with that in normal individuals. These results suggest that increased adhesion to fibronectin as well as to CS-1A oligopeptides by $\mathrm{T}$ cells from the patients with vasculitis can be ascribed to the increased function of VLA-4. Moreover, we showed that anti-VLA-4 antibody almost completely inhibited this adhesion (data not shown), confirming the above results.

Clinical course of the representative patients and the expression and function of integrin adhesive receptors. We found upregulated expression and function of these integrin adhesive receptors in the SLE patient with vasculitis. The next question is whether such alteration of the integrin receptors may be correlated with the clinical course of the disease. To address this question, we examined for expression of integrin adhesive receptors on the PBL from patients with vasculitis whom we could follow up on serially. As shown in the left panel of Fig. 5,

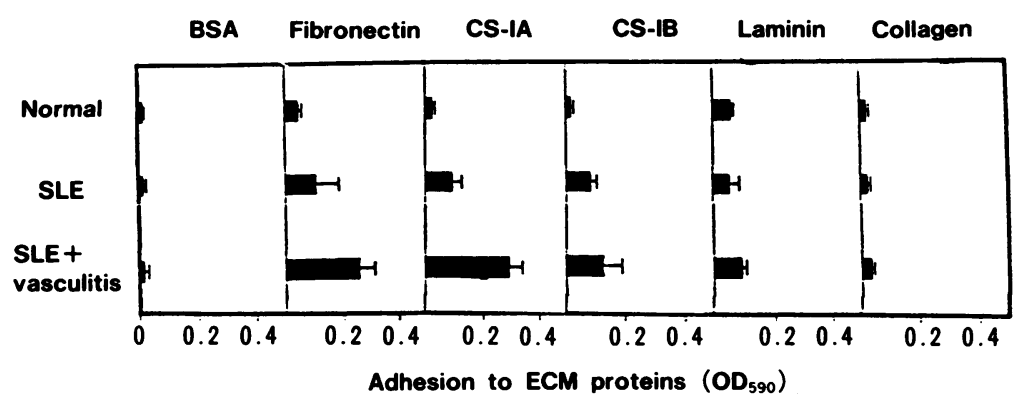

Figure 3. Adhesion of peripheral blood T cells from sexand age-matched normal individuals $(n=27)$, SLE patients without vasculitis $(n=21)$, and those with vasculitis $(n=6)$ to a series of extracellular matrix proteins and synthetic oligopeptides corresponding to the VLA-4 binding region was examined. Adherent cells were fixed with formaldehyde, stained with trypan blue, and lysed with $10 \%$ SDS as described in the Methods. Results were expressed as $\mathrm{OD}_{590}$ of individual wells. 


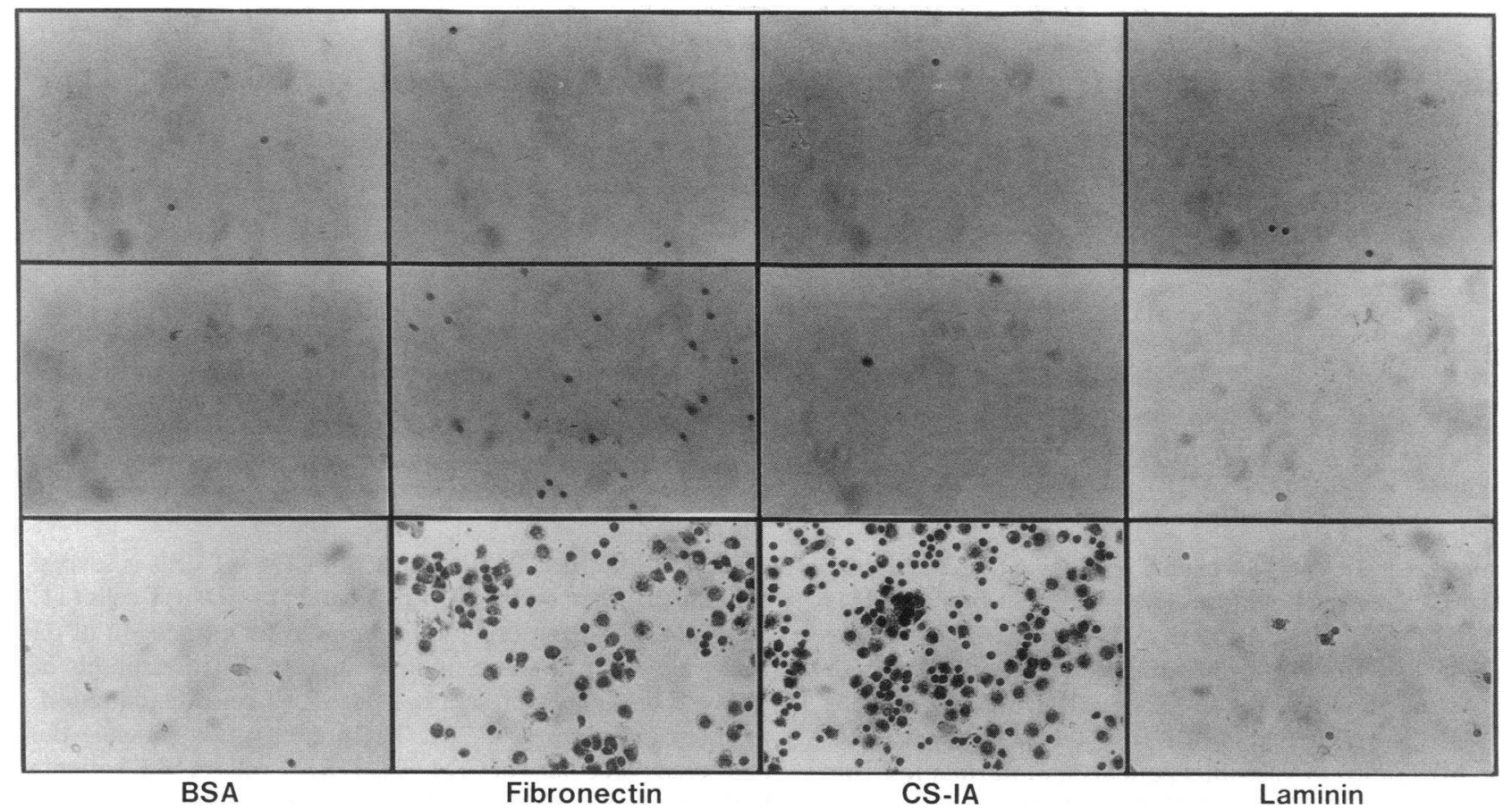

Figure 4. Adhesion of peripheral blood T cells from a normal individual ( upper column), a SLE patient without vasculitis (middle column), and that with vasculitis (lower column) to negative control (BSA), fibronectin, CS-1A oligopeptides, and laminin was shown. After adhesion to the plates, the adherent cells were fixed with formaldehyde, and stained with trypan blue. Photography was taken at 1:100.

the MFI of VLA-4 (closed circle) was significantly elevated in patient F.S. Soon after that, she experienced digital necrosis of the left fourth finger and was admitted to the hospital. She was successfully treated with increased doses of prednisolone and surgical debridement of the necrotic finger, and expression of VLA-4 returned to within normal limits. Also in patient Y.K., expression of VLA-4 and the percentage of the CD29 high population (open circles) were increased when she experienced a digital infarct due to necrotizing vasculitis, whereas expression of these molecules returned to normal in July 1992. However, when digital necrosis recurred shortly after that, the MFI of the VLA-4 antigen increased again. Although the sample size is rather small and we may have to wait to draw definitive conclusions from these two cases, the correlation of upregulated expression of VLA-4 antigen and the vasculitic events observed here further support the idea that such adhesive receptors may participate in the pathogenesis of vasculitis in patients with SLE.

Functional epitopes of the VLA-4 antigen in SLE patients with vasculitis. The above results suggest that upregulated expression and function of VLA-4 occurs on PBL from the SLE patients with vasculitis, and it has been reported that there are three distinct and independently inhibitable functional epitopes on VLA-4 molecules (22). Since the increased expression of VLA-4 obtained in the above study was derived from MFI by SM-27, it remains to be determined whether epitopes other than that recognized by SM-27 are upregulated or not. To test this, we used a series of anti-VLA-4 mAbs and examined PBL from the vasculitis patients for expression of the distinct functional epitopes. Since SM-27 could block the aggregation induced by L- 25 defining the B2 epitope, adhesion to CS-1

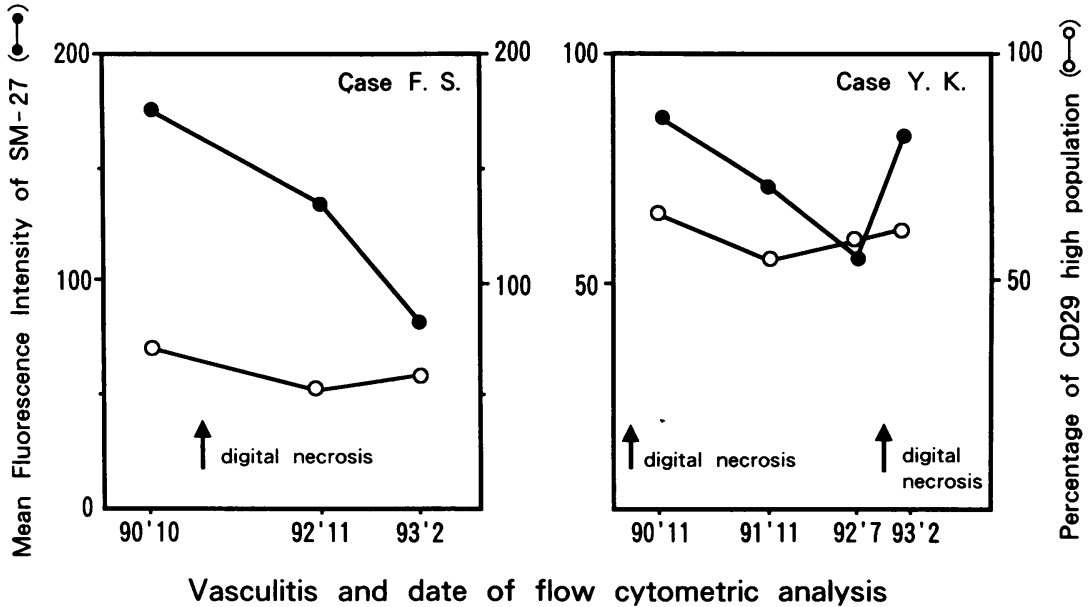

Figure 5. Time course of the expression of VLA-4 alpha and beta chains in the two representative SLE patients complicated by vasculitis. Percent expression of CD29 (VLA-4 beta chain) high + population was shown as open circles. 


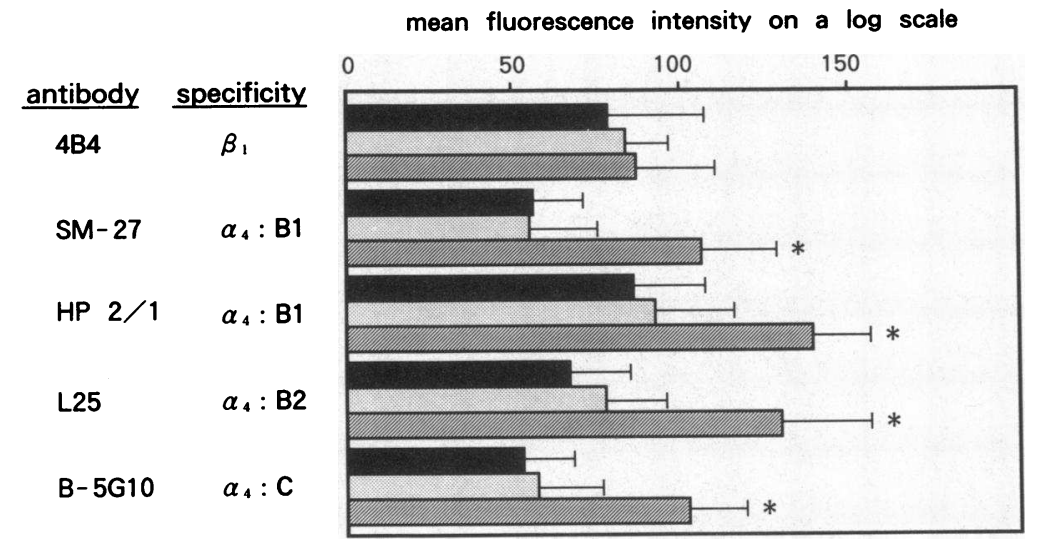

Figure 6. MFI of functional epitopes on the VLA-4 alpha chain recognized by various anti-VLA-4 antibodies expressed on PBL from sex- and age-matched normal individuals ( lack bar; $n=27$ ), SLE patients without vasculitis (gray bar; $n=21$ ), and those with vasculitis (shaded bar; $n=6$ ) was shown. ${ }^{*} P<0.05$. of fibronectin and to VCAM-1 (data not shown), SM-27 seems to define B1 functional epitopes. Using HP2/1 (defines B1), SM-27 (B1), L-25 (B2), B-5G10 (C), we observed a similar increase in the MFI of all the epitopes recognized by these mAbs. As shown in Fig. 6, the MFI of HP2/1, SM-27, L-25, and B-5G10 was increased in PBL from the vasculitis patients and the MFI ratios of vasculitis patients and normal individuals are $1.75,1.72,1.77$, and 1.67 , respectively.

These results suggest that the increase in MFI of VLA-4 antigen did not result from an increase of one particular functional epitope but was due to an increase in the number of VLA-4 molecules expressed on PBL.

Structure of the VLA-4 antigen in SLE patients with vasculitis. To further examine the structural basis of VLA-4 molecules expressed on PBL, we carried out an immunoprecipitation analysis of the VLA-4 antigen on PBL from these patients. As seen in Fig. 7, we clearly showed 140-kD bands corresponding to the intact $\alpha_{4}$ chain of VLA-4 antigen as well as 80- and $70-\mathrm{kD}$ fragments, from normal individuals, the SLE patients without vasculitis and those with vasculitis. The $80-$ and $70-\mathrm{kD}$ bands had more intense signals in the patients with vasculitis than those of normal individuals and the patients without vasculitis, supporting the above observations that the number of VLA-4 molecules expressed on PBL in the SLE patients with vasculitis was greater than those of normal individuals and SLE patients without vasculitis. Surprisingly, we observed an extra band migrating around $180 \mathrm{kD}$ along with the $80-, 70-$, and $140-\mathrm{kD} \alpha_{4}$ bands in three of the six patients with vasculitis.

\section{Discussion}

In this study, we demonstrated upregulation in expression and function of $\beta 1$ as well as $\beta 2$ integrins on PBL from SLE patients with vasculitis. More specifically, expression of VLA-4 and LFA- 1 was significantly greater in patients with vasculitis than in normal individuals, but LFA-1 was also upregulated in SLE patients without vasculitis. Function in terms of adhesiveness to cytokine-activated endothelial cells and the CS-1 domain of fibronectin was enhanced in SLE patients with vasculitis. Moreover, among those patients with upregulation of integrin adhesive receptors, half of the patients with vasculitis had the high molecular mass form (180-kD) of VLA-4.

It is well accepted that CD4+ T cells are distinguished by their differential expression of isoforms of CD45, namely CD45RA and CD45RO, with distinct functions (23-25). In addition, it has been shown that expression of the $\beta 1$ and of $\alpha_{4}$, $\alpha_{5}$, and $\alpha_{6}$ on the CD45RO subset of CD4+ T cells is several times higher than on the CD45RA subset of CD4+ T cells ( 11 , 21 ). Similar observations were reported for expression of $\beta 2$ integrins $(9,18)$. These findings suggest that the subsets of CD4 + $T$ cells may represent two stages of $T$ cell differentiation, memory and naive (26-28). The present study showing the decreased percentage of CD4+CD45RA + cells in SLE patients both with and without vasculitis supports the previous observations $(16,23)$. One may wonder whether the increased expression of these adhesion molecules could be the result of accelerated conversion from CD45RA to CD45RO in these vasculitic patients. However, the increased expression of VLA-4 antigen was observed only in the patients with vasculitis, not in the patients without vasculitis, while both kinds of patients had a

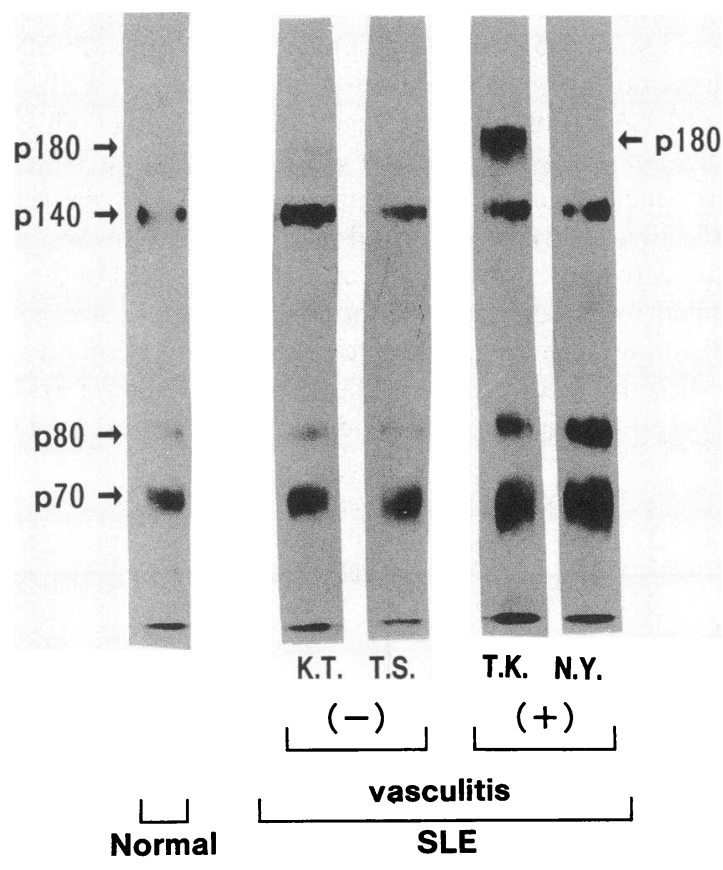

Figure 7. Immunoprecipitation analysis of VLA-4 expressed on PBL from a normal individual, two representative cases of SLE patients without vasculitis, and those with vasculitis. VLA-4 antigen was immunoprecipitated by SM-27 and protein G-sepharose beads, run on $6 \%$ homogeneous acrylamide gels. After electrophoresis, the proteins were blotted onto PVDF membranes and detected by chemiluminescence-enhancing reagents as described. 
decrease in CD4+CD45RA + cells with a normal level of VLA5 , which argues against this possibility. The pattern of expression in $\beta_{1}$ integrins in which $\alpha^{4}$ is increased, but $\alpha^{5}$ is normal has also been found in synovial $\mathrm{T}$ cells in patients with rheumatoid arthritis (29). Such differential expression of $\alpha^{4}$ and $\alpha^{5}$ has been demonstrated in human PBL after activation with ConA and PMA (30). Given the evidence that the structure of the $\alpha^{4}$ gene promoter is quite different from that of the $\alpha^{5}$ gene (31, 32 ), the differential expression of integrins in the SLE patients with vasculitis could be determined at the message level.

The constitutive expression of integrins does not necessarily indicate that they are functionally active $(9,11,21)$. Accordingly, we carried out functional studies to determine the adhesive function of these molecules, and found that VLA-4 and LFA-1 were functionally upregulated as well. The study of inhibition by various anti-VLA-4 antibodies showing that enhanced adhesion of the patients' $T$ cells to the CS-1 domain of fibronectin was completely inhibited by anti-VLA-4 antibodies, indicates that VLA-4 has the major receptors for binding to the CS-1 domain of fibronectin in these patients. On the other hand, enhanced adhesion of the patients' $T$ cells to cytokineactivated endothelial cells was inhibited $\sim 80 \%$ by a combination of anti-VCAM-1 and anti-ICAM-1. Thus, the results indicate an alternative adhesion pathway in this adhesion system. Since other pathways such as CD44/hyaluronic acid and carbohydrates/ELAM-1 are postulated to involve adhesion to endothelial cells (33-35), the possibility should be examined in the future. Nevertheless, VLA-4/VCAM-1 and LFA-1/ ICAM-1 interactions are main contributors to the enhanced adhesion to cytokine-activated endothelial cells. Since it has been demonstrated that LFA-1 / ICAM-1 and VLA-4/VCAM1 pathways have different roles in adhesion and transendothelial migration of $T$ cells (36), upregulated function of both pathways may be required to induce vascular inflammation.

Upregulation of LFA- 1 expression was also observed in patients with vasculitis as well as those without vasculitis, suggesting that VLA-4, in addition to increasing the expression of LFA-1, seems to have a key role in the pathogenesis of vasculitis. Therefore, we focused on the VLA-4 molecule and delineated further detailed mechanisms for the increased expression and function of VLA-4.

The immunoprecipitation of the VLA-4 antigen from the peripheral blood lymphocytes showed that the 80-, and 70-kd fragments of $\alpha^{4}$ occurred with more intensity in the SLE patients with vasculitis than normal individuals and those without vasculitis. These results further support the evidence obtained by indirect immunofluorescence analysis, using a variety of anti-VLA 4 antibodies recognizing different functional epitopes of the $\alpha^{4}$ chain. Several lines of evidences, therefore, suggest the upregulated expression of VLA-4 molecules, but not of a particular epitope of the $\alpha^{4}$ chain. Surprisingly, the high molecular mass bands were identified in three of the six SLE patients with vasculitis. We propose two possible sources of this $180-\mathrm{kD}$ band: $(a)$ the high molecular mass form of the VLA-4 $\alpha$ chain, and ( $b$ ) another molecule associated with VLA-4. Since we used conditions for preparing the cell lysate in which the $\alpha \beta$ heterodimer dissociates, it is less likely that the $180-\mathrm{kD}$ band is the other $\alpha$ chain of VLA, for example $\alpha^{2} . \alpha_{\text {IEL }}$ with molecular mass of $180-\mathrm{kD}$ has been shown to associate with $\beta 7$ and display an adhesion function similar to that of VLA-4. However, we did not observe the expression of HML-1 antigen on PBL from the patients with vasculitis, which argues against that possibility. These results, therefore, favor the former possibility. In this regard, Parker et al. recently described a novel form of the integrin $\alpha^{4}$ subunit with a molecular mass of $180-\mathrm{kD}$ on the PEER T cell line, and demonstrated that $\alpha^{4 / 180}$ and $\alpha^{4 / 150}$ represent different conformations of the same $\alpha^{4}$ polypeptide (37). Although the function and significance of $\alpha^{4 / 180}$ awaits further investigation, study is now in progress to determine whether the $180-\mathrm{kD}$ band is $\alpha^{4 / 180}$ or not.

Fauci reported that many patients with active vasculitis do not have demonstrable circulating or deposited immune complexes (38), and Savage stated that the mere presence of immune deposits does not necessarily result in tissue injury with an inflammatory infiltrate and fibrinoid necrosis, since deposits may be demonstrated in vessel walls without accompanying vasculitic lesions (6). It has been suggested that an alternative mechanism such as the Schwartzman reaction, but not the Arthus reaction, participates in the pathogenesis of vasculitis (7). In this model, intravascular LFA-1 / ICAM-1 (leukocyteendothelium) adhesion was shown to be necessary for this type of cytokine-primed, neutrophil-dependent vasculitis to occur (8). Given the evidence that VLA-4 mediates homotypic aggregation of lymphocytes, and adhesion of lymphocytes to endothelial cells via VCAM-1 molecules as well as to the alternatively spliced domain of fibronectin $(21,39,40)$, the upregulated function of VLA-4 in addition to that of LFA-1 could be the mechanisms responsible for the development of the characteristic pathological features of vasculitis in SLE. Furthermore, as suggested in the RA synovial T lymphocytes $(13,29,41)$, VLA-4/VCAM-1 and LFA-1 / ICAM-1 pathways not only mediate adhesion of leukocyte-leukocyte and leukocyte-endothelium, but by delivering signals into the cells to proliferate by interacting with their ligands could be important mechanisms for the infiltration of the inflammatory cells into the vessel walls and interstitium, and the maintenance of these cells to perpetuate the vascular inflammation.

The present study provides the first evidence that expression and function of VLA-4 antigen was upregulated in SLE patients with vasculitis, introducing a potential role of VLA-4 antigen in the pathogenesis of vasculitis in SLE. At present, however, it is not clear whether the upregulated expression of integrins is the consequence of the vasculitis events or is directly involved in the pathogenesis. To address the issue, it may be useful to examine the effect of anti-VLA-4 antibody in model animals for vasculitis. It has been reported that induction of adjuvant arthritis in Lewis rats could be successfully inhibited by anti-ICAM-1 antibody (42) and rat cardiac allograft survival was extended by administration of anti-ICAM-1 and/or anti-LFA-1 antibody in heterotropic rat cardiac transplantation (43). If it is the case in model animals for vasculitis by anti-VLA-4 antibody, the intervention with the VLA-4 pathway in addition to LFA-1/ICAM-1 interaction may contribute to developing a new and ideal therapeutic strategy for the intractability and sometimes fatality of SLE caused by vasculitis.

\section{Acknowledgments}

We would like to thank Drs. Martin E. Hemler and Takami Matsuyama for valuable discussions.

This study was supported in part by a grant-in-aid for scientific research $(C)$ from the Ministry of Education, Science and Culture, Japan. 


\section{References}

1. Tan, E. M. 1989. Diagnostic markers for autoimmune disease and probes for cell biology. Adv. Immunol. 14:93-151.

2. Ebling, F. M., and B. H. Hahn. 1989. Pathogenic subsets of antibodies to

DNA. Int. Rev. Immunol. 5:79-95.

3. Tan, E. M. 1991. Autoantibodies in pathology and cell biology. Cell. 67:841-842.

4. Dixon, F. J., J. D. Feldman, and J. J. Vazquez. 1961. Experimental glomerulonephritis: the pathogenesis of a laboratory model resembling the spectrum of human glomerulonephritis. J. Exp. Med. 113:899-920.

5. Winfield, J. B., I. Faiferman, and D. Koffler. 1977. Avidity of anti-DNA antibodies in serum and IgG glomerular eluates from patients with SLE. J. Clin. Invest. 59:90-96.

6. Savage, C. O. S. 1991. Pathogenesis of systemic vasculitis. In Systemic Vasculitides. A. Churg, J. Churg, and G. G. Hunder, editors. Igaku Shoin, New York. 7-30.

7. Philips, M. R., S. B. Abramson, and G. Weissman. 1989. Neutrophil adhesion and autoimmune vascular injury. Clin. Asp. Autoimmunity. 3:6-15.

8. Argenbright, L. W., and R. W. Barton. 1992. Interactions of leukocyte integrins with intercellular adhesion molecule 1 in the production of inflammatory vascular injury in vivo. J. Clin. Invest. 89:259-272.

9. Springer, T. A. 1990. Adhesion receptors of the immune system. Nature (Lond.). 346:425-434.

10. Hynes, R. O. 1992. Integrins: versatility, modulation, and signaling in cell adhesion. Cell. 69:11-25.

11. Shimizu, Y., G. A. Van Seventer, K. J. Horgan, and S. Shaw. 1990. Regulated expression and binding of three VLA (B1) integrin receptors on T cells. Nature (Lond.). 345:250-253.

12. Cybulsky, M. I., and J. Gimbrone M. A. 1990. Endothelial expression of a mononuclear leukocyte adhesion molecule during atherogenesis. Science (Wash. DC). 251:788-791.

13. Laffon, A., V. R. Garcia, A. Humbria, A. A. Postigo, A. L. Corbi, L. M. de and M. F. Sanchez. 1991. Upregulated expression and function of VLA-4 fibronectin receptors on human activated $\mathrm{T}$ cells in rheumatoid arthritis. J. Clin. Invest. 88:546-52.

14. Takeuchi, T., and T. Abe. 1993. Possible role of VLA-4 antigen in vasculitis of the patients with systemic lupus erythematosus (SLE). In Vasculitis Syndromes. T. Tanaber, editor. Hokkaido University Press, Sapporo. 187-196.

15. Tan, E. M., A. S. Cohen, J. F. Fries, A. T. Masi, D. J. McShane, N. F. Rothfield, J. G. Schaller, N. Talal, and R. J. Winchester. 1982. The 1982 revised criteria for the classification of systemic lupus erythematosus. Arthritis Rheum. 25:1271-1277.

16. Takeuchi, T., S. Tanaka, A. D. Steinberg, T. Matsuyama, J. Daley, S. F. Schlossman, and C. Morimoto. 1988. Defective expression of the $2 \mathrm{H} 4$ molecule after autologous mixed lymphocyte reaction activation in systemic lupus erythematosus patients. J. Clin. Invest. 82:1288-1294.

17. Takeuchi, T., C. E. Rudd, S. F. Schlossman, and C. Morimoto. 1987. Induction of suppression following autologous mixed lymphocyte reaction: role of a novel $2 \mathrm{H} 4$ antigen. Eur. J. Immunol. 17:97-103.

18. Sanders, M. E., M. W. Makgoba, S. O. Sharrow, D. Stephany, T. A. Springer, H. A. Young, and S. Shaw. 1988. Human memory T lymphocytes express increased levels of three cell adhesion molecules (LFA-3, CD2, and LFA1) and three other molecules (UCHL-1, CDw29, and Pgp-1) and have enhanced IFN- $\gamma$ production. J. Immunol. 140:1401-1407.

19. Pober, J. S., and R. S. Cotran. 1990. The role of endothelial cells in inflammation. Transplantation (Baltimore). 50:537-544.

20. Carlos, T. M., R. B. Schwartz, N. L. Kovach, E. Yee, M. Rosso, L. Osborn, G. Chi-Rosso, B. Newman, R. Lobb, and J. M. Harlan. 1990. Vascular cell adhesion molecule-1 mediates lymphocyte adherence to cytokine-activated cultured human endothelial cells. Blood. 76:965-970.

21. Hemler, M. E., M. J. Elices, C. Parker, and S. Takada. 1990. Structure of the integrin VLA-4 and its cell-cell and cell-matrix adhesion. Immunol. Rev. 114:45-65.

22. Pulido, R., M. J. Elices, M. R. Campanero, L. Osborn, S. Schiffer, P. A. Garcia, R. Lobb, M. E. Hemler, and M. F. Sanchez. 1991. Functional evidence for three distinct and independently inhibitable adhesion activities mediated by the human integrin VLA-4. Correlation with distinct alpha 4 epitopes. J. Biol. Chem. 266:10241-10245.

23. Morimoto, C., A. D. Steinberg, N. L. Letvin, M. Hagan, T. Takeuchi, J. Daley, H. Levin, and S. F. Schlossman. 1987. A defect of immunoregulatory T cell subsets in systemic lupus erythematosus patients demonstrated with anti2H4 antibody. J. Clin. Invest. 79:762-768.

24. Smith, S. H., M. H. Brown, D. Rowe, R. E. Callard, and P. C. Beverley 1986. Functional subsets of human helper-inducer cells defined by a new monoclonal antibody, UCHL-1. Immunology. 63:58-62.

25. Thomas, M. L., and L. Lefrancois. 1988. Differential expression of the leukocyte-common antigen family. Immun. Today. 9:320-326.

26. Akbar, A. N., L. Terry, A. Timms, P. C. L. Beverley, and G. Janossy. 1988 Loss of CD45R and gain of UCHL-1 reactivity is a feature of promed T cells. $J$. Immunol. 140:2171-2178.

27. Serra, H. M., J. F. Krowka, J. A. Ledbetter, and L. M. Pilarsky. 1988. Loss of CD45R (Lp220) represents a post-thymic T cell differentiation event. J. Im munol. 140:1435-1441

28. Sanders, M. E., M. W. Makgoba, and S. Shaw. 1988. Human naive and memory T cells: reinterpretation of helper-inducer and suppressor-inducer subsets. Immunol. Today. 9:195-199.

29. Garcia-Vicuna, R., A. Humbria, A. A. Postigo, C. Lopez-Elzaurdia, M. O. De Landazuri, F. Sanchez-Madrid, and A. Laffon. 1992. VLA family in rheumatoid arthritis: evidence for in vivo regulated adhesion of synovial fluid $\mathrm{T}$ cells to fibronectin through VLA-5 integrin. Clin. Exp. Immunol. 88:435-441.

30. Kohn, F. R., M. E. Grigg, and H.-G. Kingemann. 1991. Differential regulation of fibronectin receptor subunit gene and cell surface expression in human peripheral blood T lymphocytes. J. Immunol. 146:1484-1489.

31. Birkenmeier, T. M., J. J. McQuillan, E. D. Boedeker, W. S. Argraves, E. Ruoslahti, and D. C. Dean. 1991. The a5bl fibronectin receptor. J. Biol. Chem. 266:20544-20549.

32. Rosen, G. D., T. M. Birkenmeier, and D. C. Dean. 1991. Characterization of the $\mathrm{a}_{4}$ integrin gene promoter. Proc. Natl. Acad. Sci. USA. 88:4094-4098.

33. Shimizu, Y., W. Newman, T. V. Gopal, K. J. Horgan, N. Graber, L. D. Beall, G. A. van Seventer, and S. Shaw. 1991. Four molecular pathways of T cell adhesion to endothelial cells: roles of LFA-1, VCAM-1, and ELAM-1 and changes in pathway hierarchy under different activation conditions. J. Cell Biol. 113:1203-1212.

34. Picker, L. J., T. K. Kishimoto, C. W. Smith, R. A. Warnock, and E. C. Butcher. 1991. ELAM-1 is an adhesion molecule for skin-homing T cells. Nature (Lond.). 349:796-799.

35. Aruffo, A., I. Stamenkovic, M. Melnick, C. B. Underhill, and B. Seed. 1990. CD44 is the principal surface receptor for hyaluronate. Cell. 61:1303-1313.

36. Oppenheimer-Marks, N., L. S. Davis, D. T. Bogue, J. Ramberg, and P. E. Lipsky. 1991. Differential utilization of ICAM-1 and VCAM-1 during the adhesion and transendothelial migration of human $\mathrm{T}$ lymphocytes. J. Immunol. 147:2913-2921.

37. Parker, C. M., C. P. Pujades, M. B. Brenner, and M. E. Hemler. 1993. $\alpha^{4 / 180}$, a novel form of the integrin $\alpha^{4}$ subunit. J. Biol. Chem. 268:7028-7035.

38. Fauci, A. S. 1987. The vasculitic syndromes. In Principles of Interna Medicine. E. Braunwald, K. Isselbacher, R. Petersdorf, J. Wilson, J. Martin, and A. S. Fauci, editors. McGraw-Hill, New York. 1438-1445

39. Bednarczyk, J. L., and B. W. McIntyre. 1990. A monoclonal antibody to VLA-4 a-chain (CDw49d) induces homotypic lymphocyte aggregation. J. Immunol. 144:777-784

40. Elices, M. J., L. Osborn, Y. Takada, C. Crouse, S. Luhowskyj, M. E. Hemler, and R. R. Lobb. 1990. VCAM-1 on activated endothelium interacts with the leukocyte integrin VLA-4 at a site distinct from the VLA-4/fibronectin binding site. Cell. 60:577-84.

41. Postigo, A. A., R. Garcia-Vicuna, F. Diaz-Gonzalez, A. G. Arroyo, M. O. De Landazuri, G. Chi-Rosso, R. R. Lobb, A. Laffon, and F. Sanchez-Madrid. 1992. Increased binding of synovial T lymphocytes from rheumatoid arthritis to endothelial-leukocyte adhesion molecule-1 (ELAM-1) and vascular cell adhesion molecule-1 (VCAM-1). J. Clin. Invest. 89:1445-1452.

42. Iigo, Y., T. Takashi, and T. Tamatani. 1991. ICAM-1 dependent pathway is critically involved in the pathogenesis of adjuvant arthritis in rats. J. Immunol. 147:4167-4171.

43. Isobe, M., H. Yagita, and K. Okumura. 1992. Specific acceptance of cardiac allograft after treatment with antibodies to ICAM-1 and LFA-1. Science (Wash. DC). 255:1125-1127. 